
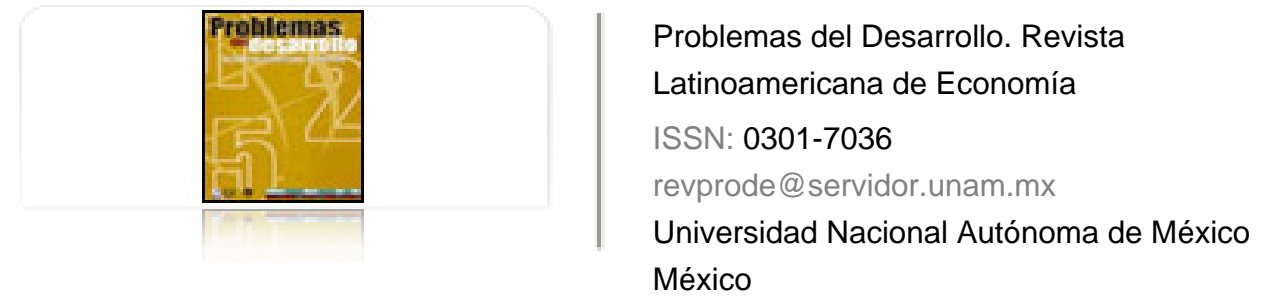

Saavedra, Francisco Leonardo

Costos del ALCA para América Latina y el Caribe: el caso de los aranceles

Problemas del Desarrollo. Revista Latinoamericana de Economía, vol. 34, núm. 133, 2003, pp. 29-48

Universidad Nacional Autónoma de México

Distrito Federal, México

Disponible en: http://www.redalyc.org/articulo.oa?id=11825949009

- Cómo citar el artículo

- Número completo

- Más información del artículo

Página de la revista en redalyc.org

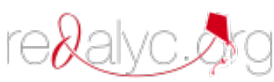

Sistema de Información Científica

Red de Revistas Científicas de América Latina, el Caribe, España y Portugal Proyecto académico sin fines de lucro, desarrollado bajo la iniciativa de acceso abierto 


\title{
Costos del alca para América latina y el Caribe: EL CASO DE LOS ARANCELES
}

\author{
Francisco Leonardo Saavedra*
}

Fecha de recepción: 18 de julio de 2003. Fecha de autorización: 23 de octubre de 2003.

\section{Resumen}

Este trabajo muestra los efectos de la apertura comercial en América Latina ( $A L$ ) y el Caribe en los sectores industrial y agrícola, particularmente en cuanto a la reducción y recomposición de la planta productiva y su efecto en los niveles de empleo, ingreso y pobreza. Nuestro propósito es mostrar que la posible puesta en práctica del Área de Libre Comercio de las Américas (ALCA) reforzaría el sentido de la apertura. También abordamos sus probables repercusiones en el sector externo, que implicarían una reestructuración de los flujos comerciales en la región y su mayor dependencia de los mercados norteamericano y canadiense.

Palabras clave: apertura comercial, sectores industrial y agrícola, sector externo, empleo, ingreso, pobreza, dependencia.

\section{Abstract}

This article demonstrates the effects from the commercial opening of Latin America and the Caribbean on industrial and agricultural sectors, particularly on the reduction and recomposition of countries' industries and their effects on levels of employment, income and poverty. The aim is to propose that the implementation of the Free Trade Area of the Americas would reinforce the effects from the opening. We will also address the likely repercussions for the export sector, which would involve a restructuring of trade flows in the region and an increased dependence on us and Canadian markets.

Key words: commercial opening, industrial and agricultural sectors, export sector, employment, income, poverty, dependence.

Doctor en Ciencias Sociales por la Universidad Autónoma Metropolitana Xochimilco. Investigador en la Universidad Pedagógica Nacional (uPN). Correo electrónico: saavedrafl@hotmail.com 


\section{Résumé}

Le présent article montre les effets de l'ouverture commerciale en Amérique Latine ( $A L$ ) et les Caraibes dans les secteurs industriel et agricole en particulier, en ce qui concerne la réduction et la recomposition de l'appareil productif et son impact sur les niveaux d'emploi, de revenu et de pauvreté. Le but de ceci est d'exposer que la possible mise en oeuvre de la Zone de Libre Echange des Amériques renforcerait le sens de l'ouverture. L'article aborde également ses répercussions probables au regard du secteur externe, celles qui impliqueraient une restructuration des flux commerciaux dans la région ainsi que la plus forte dépendance des marchés Amérique du Nord et Canada.

Mots-cléfs: ouverture commerciale, secteurs industriel et agricole, seteur externe, emploi, revenu, pauvreté, dépendance.

\section{Resumo}

Este artigo mostra os efeitos da abertura comercial na América Latina (AL) e no Caribe nos setores industrial e agrícola, principalmente com relação à redução e recomposição da planta produtiva e o seu efeito a níveis de emprego, renda e pobreza. O propósito é expor que a possível colocação em prática da Área de Livre Comércio das Américas (ALCA) reforçaria o sentido da abertura. Também coloca as prováveis repercussões no setor externo que implicariam numa reestruturação dos fluxos comerciais na região e a sua maior dependência dos mercados norte-americano e canadense.

Palavras chave: abertura comercial, setores industrial e agrícola, setor externo, emprego, renda, pobreza, dependência 


\section{Introducción}

$\mathrm{D}$ esde que la Iniciativa de las Américas en 1994 planteó la posibilidad de crear el Área de Libre Comercio para el hemisferio occidental, con el propósito de establecer un "Pacto para el desarrollo y la prosperidad: democracia, libre comercio y desarrollo sostenible en las Américas", se abrió un amplio debate sobre los beneficios que podría generar un acuerdo de integración de estas dimensiones, particularmente para los países latinoamericanos. La discusión ha tenido como eje las notables asimetrías entre las economías del continente y, en consecuencia, la vulnerabilidad de sus aparatos productivos, especialmente de las más pequeñas.

Este artículo tiene como objetivo examinar los costos de la apertura comercial que implicaría el Área de Libre Comercio de las Américas (ALCA) para las economías de los países latinoamericanos y caribeños, a partir del estado actual de las negociaciones, de manera particular, sobre el abatimiento arancelario en los sectores industrial, agrícola y exportador. Su estructura comprende cinco partes: la primera destaca los avances en la discusión del documento rector que normaría dicho acuerdo. La segunda explora esas consecuencias en la industria. La tercera refiere la problemática del sector agrícola. La cuarta describe las modificaciones del sector exportador frente a la previsible puesta en vigor del acuerdo del área comercial y, finalmente, se exponen las conclusiones.

\section{Marco jurídico}

Los resultados de la discusión para el establecimiento de la zona de libre comercio que representa la propuesta del ALCA se pueden observar en el segundo borrador del futuro acuerdo presentado en noviembre de 2002. En principio, de su lectura se desprenden las siguientes observaciones generales: primero, registra avances muy importantes en el diseño del acuerdo. Segundo, existe continuidad en los compromisos que han establecido los países del continente en el marco de los organismos multilaterales, acuerdos regionales y los de carácter bilateral, y tercero, en relación con lo anterior, regula de manera puntual el intercambio de bienes, servicios y capitales. La temática que aborda es amplia (agricultura, compras del sector público, inversión, acceso a mercados, subsidios, antidumping, derechos compensatorios, solución de controversias, servicios, derechos de propiedad intelectual, política de competencia y temas generales o institucionales), sin embargo, en 
este artículo solamente se aborda lo relacionado con las medidas arancelarias que pertenecen al capítulo sobre acceso a mercados con relación a la industria y la agricultura.

Dentro del aspecto arancelario deseamos destacar que el borrador que aquí se comenta, en su artículo 3, se beneficia con los avances en los acuerdos establecidos entre países del continente, especialmente de los subregionales que parten, en su mayoría, de condiciones más o menos semejantes. Es decir, no se toman en cuenta las grandes diferencias entre los bloques de países, aunque esto último se matiza a través de la disposición 4.10 que otorga plazos mayores, diferenciados y de gracia para la desgravación de las economías pequeñas. Un aspecto preocupante es el relacionado con la intención de institucionalizar el neoproteccionismo en su artículo 16.1 que establecería la posibilidad de restringir o prohibir la importación o exportación de bienes "por razones de seguridad nacional, salud pública, preservación de la flora o fauna, del ambiente, sanitarias y fitosanitarias, normas, etiquetas, reglamentos técnicos, compromisos internacionales, requerimientos de orden público o cualquier otras regulaciones" que se introducen como medidas no arancelarias.

El artículo 4.4 destaca el compromiso de las partes integrantes del acuerdo para que, al momento de entrar en vigor, se inicie el proceso de desgravación arancelaria mediante un calendario aún no definido. Éste es un aspecto central en la medida que las economías mayores, fundamentalmente la norteamericana, no tendrán que hacer un sacrificio especial por tener bajas tasas arancelarias. En cambio, para las economías pequeñas representará un esfuerzo de carácter estructural, porque su ingreso público depende en alto grado de sus actuales tasas arancelarias, que en su mayoría son elevadas.

Por otro lado, cabe señalar que en el capítulo sobre agricultura, la redacción del borrador es reiterativa sobre la eliminación arancelaria. Dice textualmente: " $6.2^{\mathrm{A}}$ Las Partes acuerdan eliminar los aranceles en su comercio recíproco entre las Partes de los productos agropecuarios originarios, según el Programa de Eliminación Arancelaria establecido en el Anexo xx. [salvo que se disponga lo contrario en este Acuerdo.]" (véase el primer borrador del ALCA en CISE, 2002). Esta disposición, seguramente será objeto de una amplia discusión dados los resultados negativos que en esta materia tuvo la reunión de la OMC de este año en Cancún, México.

Una conclusión de lo expuesto sobre el borrador del ALCA es que este documento parece constituir una extensión de los planteamientos de otros mecanismos como el TLCAN y el ALCEU. Por lo tanto, si la redacción final no sufre transformaciones importantes que atiendan más a las condiciones de disparidades y asimetrías en el continente, este instrumento de integración posiblemente ampliará las ya existentes.

\section{La apertura de las economías}

La recesión económica que se presentó en los países desarrollados en los primeros años de la década de los ochenta y su coincidencia con la crisis de las economías latinoamericanas 
enmarcaron una serie de reformas estructurales al modelo de desarrollo de éstas últimas, el cual se había basado, principalmente en el esquema de sustitución de importaciones. ${ }^{1}$ Así, entre 1980 y 1983 el PIB de los países desarrollados fue menor a 1\% como promedio anual; la tasa de inversión decreció en los primeros cuatro años de la década y el crecimiento del comercio mundial se contrajo entre 1980 y 1983 (Estévez, 1985). En Latinoamérica y el Caribe el inicio de la década de los ochenta fue el principio de la llamada década perdida porque al finalizar ésta, la renta per cápita era menor de lo que había sido al principio (Yergin y Stanislaw, 1999:357). La crisis que se precipitó en 1981 fue descrita por la CEPAL (1985) como "la más profunda y prolongada desde la crisis mundial de los años treinta". Otras de sus manifestaciones fueron: la caída del producto, que en 1981 fue de sólo $1.2 \%$, y entre 1983 y 1990 la evolución del PIB apenas fue de 1.7\% como promedio anual (Grien, 1994:510 y Villarreal, 1983); la inflación llegó a muy altos niveles con cifras de dos, tres y cuatro dígitos. ${ }^{2}$ Pero sin duda, el problema latinoamericano de mayor relieve en el ámbito mundial fue el de la deuda pública de largo plazo, cuya cantidad casi se cuadruplicó entre 1975 y 1982, de 45,200 a 176,400 millones de dólares. Si a esto se le suman los préstamos a corto plazo y los créditos del FMI, en 1982 ascendía a 333 mil millones de dólares (Yergin y Stanislaw, 1999: 356). De hecho la crisis de la deuda pública se desencadenó cuando en agosto de 1982 el gobierno mexicano declaró la posibilidad de suspender su pago.

En este marco surgió un proceso unilateral de apertura de mercados en Latinoamérica y el Caribe, buscando impulsar un cambio definitivo a largos años de proteccionismo y de aplicación del modelo de sustitución de importaciones. ${ }^{3}$ La apertura se dio, principalmente,

Este modelo se aplicó en América Latina y el Caribe, esencialmente, a partir de la Segunda Guerra mun dial. Ésta impuso a los países latinoamericanos, desde la perspectiva del desenvolvimiento económico, una do ble realidad: por un lado, modificó el entorno del comercio mundial, con sus obvias repercusiones en la re gión. Por otro, estimuló una mayor administración del crecimiento económico, especialmente del industrial. La mayor administración del crecimiento económico se hizo necesaria, porque con la guerra disminuyeron los flujos comerciales de bienes manufacturados provenientes de los países de gran desarrollo, lo que se tradujo en una obligada continuación de la política de sustitución de importaciones. El Estado, promotor del desarrollo a partir de los años 30, consolidó su función rectora del crecimiento económico a través de la inversión en infraestructura, esquemas fiscales favorables al capital industrial, precios bajos de materias primas y de los bienes y servicios producidos por las empresas estatales, así como de un mayor proteccionismo al aumentar las medidas arancelarias y paraarapncelarias. Es importante señalar que la querra fue la oportunidad para que los países de América Latina que no habían iniciado procesos de industrialización lo hicieran (Sunkel y Paz, 1978). Por su parte, Ferrer (2001:72) subraya tres consecuencias en la aplicación de este modelo: no generó ventajas competitivas suficientes, a esto se debe que la participación de América Latina en el comercio mun dial haya disminuido de $14 \%$ en 1945 a 5\% en 1970. La presencia de empresas filiales extranjeras con amplio control del desarrollo tecnológico debilitó "la capacidad de integrar la producción de bienes y servicios con los sistemas nacionales de ciencia y tecnología y con la oferta interna de insumos complejos". Finalmente, la debilidad del comercio externo de estos países se agravó por los desequilibrios fiscales y el endeudamiento público.

2 Los porcentajes anuales de la inflación en América Latina entre 1982 y 1990 fueron: 1982 (84.6); 1983 (130.5); 1984 (184.7); 1985 (274.1); 1986 (64.5); 1987 (198.5); 1988 (778.8); 1989 (1,201.0) y 1990 $(1,185.0)$ (Grien, 1994:512).

3 De 1985 a 1995 se instrumentaron reformas en veinte paises latinoamericanos y del Caribe, como se señala a continuación: 1985, Bolivia y Chile; 1987, Jamaica; 1988, El Salvador, Guatemala y México; 1989, Paraguay y Venezuela; 1990, República Dominicana, Honduras y Perú; 1991, Argentina, Colombia y Nicaragua; 1992, Ecuador y Guyana; 1993, Trinidad y Tobago; 1994, Brasil; y 1995, Haití y Suriname. 
a partir de un progresivo abatimiento arancelario y de un nuevo impulso a la administración pactada del comercio intraregional. Con relación a este último aspecto, la Asociación Latinoamericana de Libre Comercio (ALALC) se transformó en Asociación Latinoamericana de Integración (ALADI) para imprimirle un mayor dinamismo al comercio. Los países caribeños se vieron obligados a establecer una amplia red de acuerdos de carácter bilateral y multilateral, entre ellos y fuera de su área, con el propósito de no quedar marginados de la tendencia general de la creación de bloques comerciales. Por ello surgieron la Comunidad del Caribe (CARICOM), la Asociación de Estados del Caribe (AEC) y la Organización de Estados del Caribe Oriental (OECO). Cabe aclarar que a través de la Iniciativa para la Cuenca del Caribe, propuesta por Estados Unidos, se han establecido acuerdos bilaterales en ese escenario. En Sudamérica surgió el mercosur y los países de América Central también iniciaron una profunda reforma comercial a partir de los años ochenta para abrir sus economías. Lo anterior les ha permitido acceder a los mercados internacionales con productos industriales, principalmente manufacturas intensivas en mano de obra como textiles y ensambles de artículos electrónicos.

El proceso de liberación arancelaria en América Latina se inició en la década de los ochenta y se aplicó de manera generalizada en los noventa, con excepción de Chile que dio principio a esta política desde 1973. La apertura fue unilateral y drástica, muy rápida en algunos países si se toman en cuenta los largos años de proteccionismo (CEPAL, 1998). A continuación veremos los casos más significativos del desarme arancelario de 12 países seleccionados. Ecuador que en 1988 tenía un arancel máximo de 325\% lo disminuyó a $35.5 \%$ a mediados de 1996 y como arancel promedio de $11.5 \%$ en 1999. Chile hasta 1973 protegía su economía con un arancel máximo de $220 \%$ y como promedio 94\%, en 1985 disminuyó el máximo a 35\% y para 1999 el arancel promedio ascendía sólo a $9.8 \%$. Bolivia, tenía un arancel máximo de $150 \%$ en 1985 y de únicamente $10 \%$ a fines de 1993 , como promedio en el primer año mencionado de 12\% llegando éste a 9.7\% en 1999. Costa Rica partió de un arancel máximo de $150 \%$ en 1986 y a fines de 1993 disminuyó a $20 \%$, su arancel promedio inicial fue de 53\% y a mediados de 1996 se abatió este porcentaje a 9\%. Venezuela inicialmente, en 1989, tenía un arancel máximo de $135 \%$ y disminuyó a fines de 1993 a 20\%, en tanto el arancel promedio del primer año mencionado fue de $35 \%$ y lo redujo a $12 \%$ en 1999. Perú, abatió su arancel máximo de $108 \%$ a $25 \%$ entre 1990 y 1993 y su arancel promedio de 66\% en el primer año referido a 13.7\% en 1999. En el caso de México, que tenía en 1985 un arancel máximo de 100\%, lo abatió a 20\% al finalizar 1993 y el arancel promedio de $24 \%$ lo redujo a $16.2 \%$ desde mediados de los ochenta a 1999 (véase Cuadro 1).

Este desarme arancelario se vio fortalecido por el abatimiento de las medidas no arancelarias. En 1985 aproximadamente la mitad del valor importado por los países centroamericanos y un tercio de los sudamericanos era afectado por alguna medida paraarancelaria. Al 
Cuadro 1

Abatimiento de aranceles en nueve países de América Latina en porcentajes

\begin{tabular}{|c|c|c|c|c|c|c|c|c|}
\hline País & $\begin{array}{c}\text { Inicio } \\
\text { del } \\
\text { programa }\end{array}$ & $\begin{array}{l}\text { Arancel } \\
\text { máximo } \\
\text { (inicial) }\end{array}$ & $\begin{array}{c}\text { Arancel } \\
\text { máximo } \\
\text { (a fines de } \\
1993 \text { ) }\end{array}$ & $\begin{array}{c}\text { Arancel } \\
\text { promedio } \\
\text { (inicial) }\end{array}$ & $\begin{array}{c}\text { Arancel } \\
\text { promedio } \\
\text { (a fines de } \\
1993 \text { ) }\end{array}$ & $\begin{array}{c}\text { Arancel } \\
\text { máximo } \\
\text { (a media- } \\
\text { dos de } \\
\text { 1996) }\end{array}$ & $\begin{array}{c}\text { Arancel } \\
\text { promedio } \\
\text { (a media- } \\
\text { dos de } \\
1996)\end{array}$ & $\begin{array}{r} \\
\text { Arancel } \\
\text { promedio } \\
\text { en } 1999\end{array}$ \\
\hline Argentina & 1989 & 65 & 30 & 39 & 15 & 33 & 14 & 13.5 \\
\hline Bolivia & 1985 & 150 & 10 & 12 & 7 & 10 & 10 & 9.7 \\
\hline Brasil & 1988 & 105 & 35 & 51 & 14 & 35 & 10 & 14.3 \\
\hline \multirow[t]{2}{*}{ Chile } & 1973 & 220 & 10 & 94 & 10 & & & 9.8 \\
\hline & 1985 & 35 & 11 & 35 & 11 & 11 & 11 & \\
\hline Colombia & 1990 & 100 & 20 & 44 & 12 & 20 & 11 & 11.6 \\
\hline Costa Rica & 1986 & 150 & 20 & 53 & 12 & 20 & 9 & $\mathrm{nd}$ \\
\hline México & 1985 & 100 & 20 & 24 & 12 & 35 & 14 & 16.2 \\
\hline Perú & 1990 & 108 & 25 & 66 & 18 & 25 & 16 & 13.7 \\
\hline Venezuela & 1989 & 135 & 20 & 35 & 10 & 20 & 12 & 12.0 \\
\hline Ecuador & 1988 & 325 & $\mathrm{nd}$ & $\mathrm{nd}$ & $\mathrm{nd}$ & 35.5 & 11.8 & 11.5 \\
\hline Paraguay & 1988 & 70 & $\mathrm{nd}$ & $\mathrm{nd}$ & $\mathrm{nd}$ & 30 & 9.5 & 11.4 \\
\hline Uruguay & 1988 & 45 & $\mathrm{nd}$ & $\mathrm{nd}$ & $\mathrm{nd}$ & 24 & 13.5 & 4.1 \\
\hline
\end{tabular}

Fuente: (CEPAL, 1998b y 2001).

finalizar 1994, en 7 países de la ALADI la proporción estaba reducida a menos de $1 \%$ y en ningún caso era superior a 5\% (Garay y Estevadeordal, $c f$. CEPAL, 2001). Ahora veremos algunos casos particulares: Argentina eliminó en gran parte las medidas no arancelarias entre 1989 y 1991, lo mismo hizo Bolivia, Colombia, Costa Rica, México, Perú y Venezuela a finales de 1990 (CEPAL, 1998b). El conjunto de esta política de apertura trajo para América Latina y el Caribe una serie de consecuencias en el desenvolvimiento industrial y en la agricultura, entre otras, incidiendo en el desempleo y en el abaratamiento del precio de la mano de obra.

\section{El caso de la industria}

En el caso de la industria, una primera observación es el desarrollo de la producción durante las últimas dos décadas, comparadas con la etapa anterior a la apertura. Así, en los años ochenta apenas creció $0.4 \%$, en los noventa lo recuperó a $3.1 \%$ como promedio anual, pero estuvo por debajo de $6.8 \%$ que alcanzó de 1950 a 1974 y aún del incremento de $4.3 \%$ entre 1974 y 1980. Este modelo de crecimiento se refleja en los datos intraindustriales. Por ejemplo, la manufacturera creció a un promedio anual de $6.3 \%$ entre 1950 y 1980 , luego se abatió a $0.2 \%$ entre 1980 y 1990 para recuperarse entre este último año y 1999 en $3.1 \%$. Algo semejante pasó en la industria de la construcción que tuvo los siguientes promedios anuales de crecimiento: $5.4 \%,-1.9 \%$ y $3.9 \%$ en los periodos señalados. Este desempeño se puede observar también en las industrias citadas pero en el marco subregional 
(CEPAL, 2001). Lo anterior revela que la etapa de instrumentación del nuevo modelo económico (durante los años ochenta) y dentro de éste, la política de abatimiento arancelario fue negativa para el sector industrial, pero la de consolidación (en los años noventa) no fue del todo positiva si la comparamos con el crecimiento de la producción durante las décadas del modelo de sustitución de importaciones.

El comportamiento de la producción aquí descrito se puede explicar en función de la profunda reestructuración de la industria en el periodo de instrumentación y consolidación de las políticas de apertura económica en la que desempeña un papel muy importante el desarme arancelario. En efecto, el primer impacto de la apertura fue el quiebre de miles de empresas, especialmente las medianas y pequeñas que no tuvieron la capacidad para enfrentar la competencia externa. A este hecho también contribuyó la eliminación de créditos preferenciales y bajas tasas de interés otorgadas por instituciones oficiales que desaparecieron en el marco de la reforma económica, principalmente en Argentina, Chile, México y Perú (Moguillansky y Bielschowsky, 2000). La apertura, por otro lado, permitió la importación de insumos y maquinaria a menores precios que antes. Parte de la industria aprovechó esta nueva circunstancia para modernizarse y aumentar su competitividad aunque a un alto costo en términos de empleo (Katz, 2000; Weller, 2000; CEPAL, 2001; Peres y Stumpo, 2002).

Algunas investigaciones sobre la desaparición de empresas asocian este hecho con la apertura de las economías latinoamericanas y arrojan los siguientes datos: en Chile quebraron unas 7,000 empresas entre 1975 y 1993, principalmente medianas; en Argentina alrededor de 15,000 en los años ochenta (Katz, 2000); en Brasil las pequeñas y medianas empresas (PYMES) representaban en 1985 16.6\% de las empresas del sector industrial y en 1994 disminuyeron su porcentaje a 13\% (Rocha y Da Silva, 2002); en Colombia, en 1985 los pequeños establecimientos representaban 71\% del total y en 1996 este porcentaje cayó a 69\%. En Uruguay se habla del "cierre de numerosas empresas" entre 1987 y 1997, en estos años el número de pequeñas empresas tuvo un crecimiento de $-7.2 \% \mathrm{y}$ el de las medianas de $-12.2 \%$ (Romaniello, 2002).

En los años noventa se modificó la composición de la producción manufacturera y se fortalecieron, por un lado, las empresas de bienes intermedios, procesadoras de recursos naturales - que incorporan un mayor porcentaje de capital y tecnología que de mano de obra-, como son: la producción de hierro, acero, productos petroquímicos, minerales no ferrosos, aceites vegetales, celulosa y papel. Por el contrario, perdieron terreno las industrias productoras de bienes de consumo final intensivas en mano de obra como calzado, vestuario, entre otras (Katz, 1999).

Sin embargo, este fenómeno ha tenido un comportamiento diferente de acuerdo al país que se trate. Por ejemplo, si se toman en consideración los años que van de 1990 a 1996 en Argentina y México, las manufacturas procesadoras de recursos naturales perdieron im- 
Cuadro 2

Cambios en el peso relativo de los sectores industriales al interior del producto manufacturero global

\begin{tabular}{|c|c|c|c|c|c|c|c|c|c|c|}
\hline \multirow[b]{2}{*}{ Sectores } & \multicolumn{2}{|c|}{ Argentina } & \multicolumn{2}{|c|}{ Brasil } & \multicolumn{2}{|c|}{ Chile } & \multicolumn{2}{|c|}{ Colombia } & \multicolumn{2}{|c|}{ México } \\
\hline & 1990 & 1996 & 1990 & 1996 & 1990 & 1996 & 1990 & 1996 & 1990 & 1996 \\
\hline I & 14.3 & 13.1 & 22.9 & 22.8 & 10.1 & 10.2 & 9.6 & 10.5 & 12.3 & 13.90 \\
\hline II & 8.5 & 12.1 & 7.0 & 8.7 & 2.3 & 2.0 & 4.3 & 6.5 & 9.5 & 10.8 \\
\hline III+IV & 46.7 & 45.7 & 39.6 & 42.4 & 55.5 & 56.2 & 51.1 & 51.2 & 46.8 & 46.5 \\
\hline V & 30.5 & 29.0 & 30.5 & 26.1 & 32.0 & 31.6 & 34.9 & 31.8 & 31.4 & 28.8 \\
\hline
\end{tabular}

I Industria metalmecánica (excluyendo automóviles)

II Equipo de transporte

III+IV Alimentos, bebidas y tabaco y IV industrias procesadoras de recursos naturales

$\mathrm{V}$ Industrias "tradicionales" intensivas en mano de obra

Fuente: Katz (1999).

portancia respecto al total del sector, en el caso del primero, en un punto porcentual y en el segundo, en tres décimas. En cambio, en Brasil, Chile y Colombia este tipo de manufacturas ganó terreno, especialmente en el primero de estos países cuyo incremento de las ramas a que hacemos referencia fue de 2.8 puntos porcentuales, mientras en Chile y Colombia fueron de siete y una décima respectivamente. Con relación a la elaboración de equipo de transporte, salvo en Chile, que presentó un retroceso relativo de 2.3 a 2.0 entre 1990 y 1996, en los otros cuatro países esta rama aumentó su importancia de 8.5 a 12.1 en Argentina; de 7.0 a 8.7 en Brasil; de 4.3 a 6.5 en Colombia y de 9.5 a 10.8 en México.

De manera opuesta, las ramas manufactureras donde la producción se realiza con base en el uso intensivo de mano de obra perdieron importancia dentro del sector. Esta pérdida de peso relativo fue en Argentina de 1.5\%; en Brasil de 4.4\%; en Chile de 0.4\%; en Colombia de $3.1 \%$ y en México de $2.6 \%$ (véase Cuadro 2).

Lo anterior se tradujo en un lento crecimiento del empleo en la industria manufacturera de la región en los años noventa. Este promedio anual apenas fue de $1.2 \%$ en 17 países seleccionados, aunque con grandes contrastes entre ellos. En cinco se dieron decrementos: Argentina $-2.2 \%$, Colombia $-0.9 \%$, Ecuador $-0.1 \%$, Perú -0.1 y Uruguay $-2.8 \%$. Por el contrario, en cuatro se observaron incrementos muy importantes: Bolivia 8\%, Honduras 8.4\%, Panamá $5.6 \%$ y Paraguay $6.2 \%$. En dos, el crecimiento fue prácticamente nulo: Brasil $0.3 \%$ y Costa Rica $0.6 \%$ y en uno, México, se dio un crecimiento intermedio $4.3 \%$, en el resto de los países creció alrededor del promedio.

Si los datos anteriores se contrastan con los de productividad, podemos afirmar que en general, entre estas dos variables existe un contraste, porque donde se dan los desempeños del empleo más negativos se observan los mayores incrementos de la productividad y por el contrario, en los países que tuvieron altas tasas de empleo, la productividad sufrió retrocesos (véase Cuadro 3). Esto nos permite afirmar que el modelo de desarrollo industrial seguido por la región dentro del modelo de economía abierta ha implicado, hasta hoy, 
Cuadro 3

Empleo manufacturero y productividad

\begin{tabular}{lccc}
\hline & Periodo & $\begin{array}{c}\text { Empleo } \\
\text { manufacturero }\end{array}$ & $\begin{array}{c}\text { Productividad } \\
\text { laboral }\end{array}$ \\
\hline \multicolumn{1}{c}{ Países grandes ${ }^{4}$} & & & \\
Argentina & $1990-1887$ & -2.2 & 7.8 \\
Brasil & $1992-1997$ & 0.3 & 4.6 \\
Colombia & $1991-1997$ & -0.9 & 2.6 \\
México & $1991-1997$ & 4.3 & -0.6 \\
Perú & $1990-1997$ & -0.1 & 5.6 \\
Venezuela & $1990-1997$ & 1.7 & 0.8 \\
$\quad$ Países medianos & & & 3.9 \\
Chile & $1990-1997$ & 2.2 & 5.7 \\
Ecuador & $1990-1995$ & -0.1 & -4.2 \\
$\quad$ Países pequeños & & & 2.8 \\
Bolivia & $1990-1997$ & 8.8 & 2.4 \\
Costa Rica & $1990-1997$ & 0.6 & -4.1 \\
El Salvador & $1992-1997$ & 2.0 & -1.5 \\
Honduras & $1990-1997$ & 8.4 & -4.9 \\
Panamá & $1991-1997$ & 5.6 & 2.7 \\
Paraguay & $1992-1997$ & 6.2 & 3.2 \\
República Dominicana & $1991-1996$ & 1.3 & -1.9 \\
Uruguay & $1990-1997$ & -2.8 & 2.3 \\
Jamaica & $1991-1996$ & 1.1 & \\
\hline$\quad$ Promedio ponderado & & 1.2 & \\
\hline
\end{tabular}

Fuente: Weller (2000).

un modesto crecimiento de la producción y de la expansión de la productividad con sacrificio del empleo (Lora y Barrera, 1998). ${ }^{5}$ Esto último combinado con el "crecimiento de la población en edad de trabajar y la económicamente activa a mayores tasas que la población total" (CEPAL, 2001) se tradujo en altas tasas de desempleo urbano y abatimiento del salario mínimo en la mayoría de los países, aspectos que se desarrollan a continuación.

\section{Desempleo urbano y niveles salariales}

El desempleo urbano se elevó entre 1990 y 1997 como promedio anual en América Latina y el Caribe a 6\%, y a $8 \%$ entre 1998 y 1999. Fue particularmente importante en Argentina donde llegó como promedio de los primeros años señalados a $11.5 \%$ y a $13.6 \%$ en los

$4 \quad$ Las definiciones de país grande, mediano y pequeño han sido abordadas por diversos organismos mundiales como la CEPAL, que basa su categorización en el número de habitantes. Así, en el primer caso se consideran a los que tienen a partir de 20 millones, en el segundo los que cuentan con un mínimo de 10 millones y los ter ceros los que están por debajo de esta última cifra.

5 A este respecto Katz (2000) afirma sobre el caso argentino: "La expansión de la productividad es la contraparte de un mayor desempleo estructural. Esta contradicción es difícil de superar, ello dependería de un buen funcionamiento de la política macroeconómica y de la credibilidad que a largo plazo los agentes económicos puedan desarrollar". 


\section{Cuadro 4}

Evolución del desempleo y salario mínimo urbanos en América Latina y el Caribe (1990-1998)

\begin{tabular}{|c|c|c|c|c|c|}
\hline $\begin{array}{l}\text { Países y } \\
\text { periodos }\end{array}$ & $\begin{array}{l}\text { Desempleo } \\
\text { urbano (tasas } \\
\text { promedio) }\end{array}$ & $\begin{array}{l}\text { Salario } \\
\text { mínimo } \\
\text { urbano }\end{array}$ & $\begin{array}{l}\text { Países y } \\
\text { periodos }\end{array}$ & $\begin{array}{l}\text { Desempleo } \\
\text { urbano (tasas } \\
\text { promedio) }\end{array}$ & $\begin{array}{c}\text { Salario } \\
\text { mínimo } \\
\text { urbano }\end{array}$ \\
\hline Argentina & & & Honduras & & \\
\hline 1990-1997 & 11.5 & 1.0 & 1990-1997 & 6.3 & 0.8 \\
\hline 1998-1999 & 13.6 & -0.7 & 1998-1999 & 5.3 & 5.1 \\
\hline Bolivia & & & México & & \\
\hline 1990-1997 & 5.2 & 6.4 & 1990-1997 & 3.8 & -5.6 \\
\hline 1998-1999 & 5.1 & 8.7 & 1998-1999 & 2.9 & -0.2 \\
\hline Brasil & & & Nicaragua & & \\
\hline $1990-1997$ & 5.1 & -1.1 & $1990-1997$ & 14.9 & $\ldots \ldots$ \\
\hline 1998-1999 & 7.6 & 3.3 & 1998-1999 & 12.0 & $\ldots .$. \\
\hline Chile & & & Panamá & & \\
\hline 1990-1997 & 7.0 & 5.5 & $1990-1997$ & 17.2 & 1.1 \\
\hline 1998-1999 & 8.1 & 4.6 & 1998-1999 & 14.6 & 0.8 \\
\hline Colombia & & & Paraguay & & \\
\hline 1990-1997 & 10.1 & -0.7 & $1990-1997$ & 5.9 & -1.3 \\
\hline 1998-1999 & 17.4 & 0.1 & 1998-1999 & 8.0 & 1.7 \\
\hline Costa Rica & & & Perú & & \\
\hline 1990-1997 & 5.3 & 0.7 & $1990-1997$ & 8.5 & 0.8 \\
\hline \multirow[t]{2}{*}{ 1998-1999 } & 5.8 & 3.6 & $1998-1999$ & 8.8 & 39.6 \\
\hline & & & República & & \\
\hline Ecuador & & & Dominicana & & \\
\hline 1990-1997 & 8.5 & 3.5 & 1990-1997 & 17.7 & 0.3 \\
\hline 1998-1999 & 13.0 & -5.4 & 1998-1999 & 14.1 & 2.8 \\
\hline El Salvador & & & Uruguay & & \\
\hline 1990-1997 & 7.9 & -1.4 & $1990-1997$ & 9.8 & -7.8 \\
\hline 1998-1999 & 7.3 & -0.6 & 1998-1999 & 10.7 & 0.7 \\
\hline Guatemala & & & Venezuela & & \\
\hline 1990-1997 & 3.8 & -13.1 & 1990-1997 & 9.6 & -3.9 \\
\hline \multirow[t]{2}{*}{ 1998-1999 } & 5.5 & -1.9 & 1998-1999 & 13.1 & -7.8 \\
\hline & & & América & & \\
\hline Haití & & & Latina & & \\
\hline 1990-1997 & $\ldots \ldots$ & -8.0 & $1990-1997$ & 6.0 & $\ldots .$. \\
\hline 1998-1999 & $\ldots \ldots$ & -12.6 & 1998-1999 & 8.4 & $\ldots .$. \\
\hline
\end{tabular}

Fuente: CEPAL (2000). 
segundos; Colombia, a $10.1 \%$ y $17.4 \%$; Ecuador, a $8 \%$ y $13 \%$; Nicaragua, a $14.9 \%$ y 12\%; Panamá, a $17.2 \%$ y $14.6 \%$; República Dominicana, a $17.7 \%$ y $14.1 \%$; Uruguay, a $9.8 \%$ y $10.7 \%$, y Venezuela, a $9 \%$ y $13.1 \%$. México fue, de los 19 países estudiados, el que mejor desempeño tuvo respecto al urbano, pues entre 1990 y 1997 su tasa se elevó a $3.8 \%$ y entre 1998 y 1999 a 2.9 (véase Cuadro 4). En dicho cuadro es posible observar la evolución de los salarios mínimos urbanos. Cinco de los diecinueve países señalados -El Salvador, Guatemala, Haití, México y Venezuela - tuvieron un crecimiento negativo durante los dos periodos citados. Destacan los casos de Guatemala y Haití por el abatimiento de este indicador, el primero con un crecimiento de $-13.1 \%$ de 1990 a 1997 y el segundo, de -12\% de 1998 a 1999. Por el contrario, siete casos —Bolivia, Chile, Costa Rica, Honduras, Panamá, República Dominicana y Perú - presentaron un crecimiento positivo. Entre estos, resaltan Bolivia, Chile y Perú, los primeros por un fortalecimiento sostenido de los salarios mínimos en la década de los noventa, que en el caso de Bolivia fue de $\mathbf{7 . 5 5 \%}$ como promedio de los dos periodos y en el de Chile de 5.05\% también como promedio en el mismo lapso. Perú, de acuerdo con esta información, resulta un caso atípico por el incremento de 39.6\% anual entre 1998 y 1999. De los seis países restantes: Argentina, Brasil, Paraguay, Colombia, Ecuador y Uruguay —en Nicaragua no hay datos-, en los tres primeros se dieron incrementos muy pequeños como promedio de los dos periodos y de los últimos tres, en dos: Colombia y Ecuador decrementos salariales también pequeños, en el último de los citados, Uruguay, un crecimiento de -3.5\% entre 1990 y 1999.

Pese a que los datos anteriores no hacen referencia a la evolución de los salarios mínimos reales (los que existen al respecto abarcan un universo muy estrecho) es posible tener una idea general del comportamiento del ingreso de la mayoría de los trabajadores de América Latina y el Caribe sujetos a las percepciones mínimas legales. Se puede observar que en promedio, incluyendo a Perú como caso atípico, su aumento en la región fue de 9.5\% como promedio anual. Si se excluye al Perú entonces se tiene una caída de $-10.55 \%$.

\section{El caso del sector agropecuario}

El cambio del modelo económico en los países de América Latina y el Caribe, al igual que en la industria, también incidió en la estructura y el desempeño del sector agropecuario. Algunos problemas que estaban presentes con anterioridad a la década de los ochenta se prolongaron y han aparecido nuevos. Aquí se abordan los relacionados directamente con la apertura de las economías precedidos de una referencia al comportamiento general de este sector.

Al hacer una comparación del crecimiento de la producción en algunas ramas en los veintiocho años que van de 1970 a 1998 vemos que el sector agropecuario ha mostrado una evolución menos que modesta. Así, el promedio de crecimiento en la década de los setenta, en veinte países seleccionados, fue $2.77 \%$; en los ochenta $1.91 \%$ y en los noventa 


\section{Cuadro 5}

America Latina y el Caribe:

evolución de la agricultura, silvicultura, caza y pesca en 19 países (tasas medias anuales y porcentajes promedio)

\begin{tabular}{lccc}
\hline \multicolumn{1}{c}{ Países grandes } & $1970-1980$ & $1980-1990$ & $1990-1998$ \\
Argentina & 2.1 & 1.6 & 4.0 \\
Brasil & 4.7 & 2.5 & 2.8 \\
Colombia & 4.4 & 3.0 & 1.3 \\
México & 3.4 & 1.1 & 1.3 \\
Perú & -0.6 & 2.2 & 4.3 \\
Venezuela & 2.9 & 2.0 & 1.2 \\
& & & $1990-1998$ \\
Países medianos & $1970-1980$ & $1980-1990$ & 4.4 \\
Chile & 2.2 & 5.7 & 2.6 \\
Ecuador & 3.0 & 4.2 & \\
& & & $1990-1998$ \\
$\quad$ Países pequeños & $1970-1980$ & $1980-1990$ & 4.0 \\
Bolivia, & 4.1 & 1.9 & 3.0 \\
Costa Rica & 2.6 & 3.1 & 0.8 \\
El salvador & 2.4 & -1.4 & 2.8 \\
Guatemala & 4.7 & 1.3 & -3.5 \\
Haití & 1.5 & -0.2 & 2.4 \\
Honduras & 2.7 & 2.7 & 4.5 \\
Nicaragua & -0.1 & -0.7 & 2.1 \\
Panamá & 1.3 & 2.5 & 2.4 \\
Paraguay & 6.7 & 4.0 & 3.6 \\
República Dominicana & 3.4 & 0.4 & 1.2 \\
Uruguay & 2.9 & 2.0 & \\
\hline
\end{tabular}

Fuente: CEPAL (2001).

2.6\% (véase Cuadro 5). Es decir, durante la década en que se realizaron las reformas económicas en la mayoría de los países, la producción sufrió una sensible disminución y en los años de consolidación de esas reformas se nota una recuperación que no llega a representar el porcentaje de los años setenta.

La tendencia general antes señalada cambia cuando se observan los casos particulares. De los países grandes sólo Argentina y Perú lograron en los noventa desempeños notables. La producción en los dos medianos, Chile y Ecuador, si bien aumentó en los años ochenta respecto a la década anterior, en los noventa decayó, aunque en el caso de Chile con un promedio de incremento anual superior al promedio general.

El desenvolvimiento agropecuario de los países pequeños ha sido muy variado, sin embargo es posible destacar algunos comportamientos generales como los observados por Guatemala, Honduras, Paraguay, Uruguay, El Salvador y Haití que tendieron al deterioro desde la década de los setenta. Este último país presenta el caso extremo pues sus promedios anuales fueron de $1.5 \%,-0.2 \%$ y $-3.5 \%$ en los respectivos periodos. De los cinco restantes, Bolivia y República Dominicana, después de una caída de la producción en los años ochenta respecto a la década anterior, en los noventa volvieron a recuperar sus pro- 
medios de crecimiento; Costa Rica, con una ligera caída en esta última década, mantuvo un promedio superior al del conjunto de los países. La producción en Panamá se desarrolló en forma zigzagueante, en los setenta con un promedio de $1.3 \%$, en los ochenta $2.5 \%$ y en los noventa $2.1 \%$. Por último, Nicaragua, con crecimientos negativos en los dos primeros periodos señalados, observó el mejor desempeño de todos en los años noventa.

La evolución aquí descrita es producto de las condiciones particulares de los países, del marco internacional en el que se ha desarrollado la región y de las reformas económicas aplicadas por los gobiernos. En esta parte se abordarán estas últimas, especialmente las relacionadas con la apertura de las economías.

En el marco general del cambio de modelo económico, la progresiva disminución de la rectoría económica del Estado impactó en el sector agropecuario. Dicho impacto se reflejó en la reducción de los apoyos oficiales a la producción y comercialización, el desmantelamiento de instituciones de crédito y de atención a los problemas sectoriales, la liberación de los precios internos, reforma o desaparición de mercados paraestatales, impulso a los mecanismos del mercado para acceder a la propiedad agrícola y la apertura económica (Ocampo, 2001; Spoor, 2001; Stallings y Peres, 2000).

A partir de esta última puede explicarse una serie de transformaciones que ha experimentado el sector agrícola en América Latina y el Caribe en la medida que permite el abaratamiento de productos, insumos y maquinaria. La competencia externa con productos subsidiados, como en el caso de Estados Unidos y la Unión Europea, ha incidido en la exclusión de pequeños productores dedicados a la explotación de ramas tradicionales orientadas al mercado interno y, al mismo tiempo, ha estimulado a grandes propietarios dedicados a ramas vinculadas al sector externo (Weller, 2000). Esto explica por qué a partir de los años ochenta se observa una mayor concentración de la tierra en pocas manos y la disminución de las unidades más pequeñas, especialmente en Bolivia, Chile, Argentina, Brasil, Colombia y México (Stallings y Peres, 2000). David, Morales y Rodríguez (2001) opinan que el grado de concentración de la tierra en muchos países de la región se mantuvo o aumentó en las últimas décadas. Estos autores afirman que, de diescisiete países estudiados a partir de la aplicación del coeficiente de Gini para la tierra, se pueden distinguir tres grupos: "el primero está integrado por Chile, México y Paraguay, cuyos índices superan el valor de 0.90. El segundo, por Argentina, Brasil, Costa Rica, El Salvador, Panamá, Perú y Venezuela, cuyos índices se sitúan entre 0.80 y 0.90. El tercer grupo está formado por Colombia, Jamaica, Puerto Rico, República Dominicana y Uruguay, con índices de entre 0.70 y 0.80 , y por Honduras, con un índice de 0.66 ”.

Por otro lado, el desarrollo del sector agropecuario también se explica en función del dinamismo de la demanda externa que observaron algunas ramas y por el comportamiento negativo que tuvieron los precios internacionales de productos tradicionales y los cambios 


\begin{tabular}{lcc} 
Cuadro 6 \\
$\begin{array}{c}\text { Cumportamiento de la producción agropecuaria en América Latina y el Caribe } \\
\text { (promedio anual de tasas de crecimiento) }\end{array}$ \\
& $\begin{array}{l}\text { 1990-1995/ } \\
\text { Productos }\end{array}$ & $1995-1999 /$ \\
$1985-1990$ & $1990-1995$ \\
\hline Cereales & 1.2 & 3.3 \\
Pecuarios & 3.8 & 4.8 \\
Oleaginosas & 0.9 & 2.7 \\
Raíces y tubérculos & 0.1 & 1.3 \\
Hortalizas & 2.2 & 3.3 \\
Frutas & 2.9 & 2.5 \\
Café verde & -0.4 & -0.7 \\
Caña de azúcar & 0.5 & 2.3 \\
\hline
\end{tabular}

Fuente: CEPAL (2001).

tecnológicos (CEPAL, 2001). Así, a escala mundial, desde la década de los años sesenta hasta los noventa, se observa un crecimiento sostenido de la demanda de hortalizas, frutas, semillas oleaginosas, tortas y harinas oleaginosas y un descenso, especialmente en los noventa, en la de cereales, raíces, tubérculos y fibras vegetales. Lo anterior se refleja, en términos generales, en el comportamiento de la producción en América Latina y el Caribe (véase Cuadro 6), en los casos en que así no sucede se debe a un comportamiento particular de la demanda interna (David, Morales y Rodríguez, 2001).

Si bien el crecimiento de la demanda mundial de productos agropecuarios estimuló las exportaciones de la región, las cuales tuvieron un incremento de $6.6 \%$ anual entre 1990 y 1997, las importaciones de estos productos asumieron un comportamiento más dinámico pues en el mismo periodo aumentaron en promedio 10.3\% (Weller, 2000). En este artículo se sugiere que esto último puede entenderse en el marco general del abatimiento arancelario de la región que, en materia de alimentos, disminuyó de 54 a 11\% en Brasil en los periodos 1984-1987 y 1991-1993, respectivamente. En Argentina de 21 a 5\%, en Bolivia de 20 a 10\%, en Chile de 20 a 11\%, en Colombia de 37 a $15 \%$ y en México aumentó de 12 a 14\%. Nótese que este comportamiento atípico tiene lugar en un país que, en el contexto de América Latina y el Caribe respecto a estos productos, tenía bajas tasas arancelarias (Spoor, 2001). Otras repercusiones de la apertura fueron el fortalecimiento de las empresas trasnacionales (en las ramas agroindustrial y de comercialización), el deterioro de los niveles de empleo y el agravamiento de la pobreza en las zonas rurales.

En el caso del empleo, de 13 países estudiados, 8 tuvieron tasas negativas de crecimiento en los años noventa: Brasil, $-1.9 \%$; Chile, $-1.6 \% \%$; Colombia, $-1.3 \%$; Costa Rica, $-0.6 \%$; El Salvador, -2.2\%; Jamaica, -2.6\%; Panamá, -2.1\% y República Dominicana, $-4.8 \%$, y en 5 países, porcentajes muy pequeños de crecimiento positivo: Ecuador $2.2 \%$; Honduras 3\%; México, 1.6\%; Paraguay, 2.2\% y Venezuela 1.3\% (Weller, 2000). 


\section{Cuadro 7}

América Latina (ocho países):

algunas características de los estratos ocupacionales, 1997*

\begin{tabular}{|c|c|c|}
\hline Estratos ocupacionales & $\begin{array}{c}\text { Porcentaje de la fuerza de } \\
\text { trabajo ocupada }\end{array}$ & $\begin{array}{c}\text { Ingreso medio (en líneas } \\
\text { de pobreza per cápita) }\end{array}$ \\
\hline 1 Empleadores & 4.3 & 15.8 \\
\hline 2 Directores, gerentes & 2.0 & 11.6 \\
\hline 3 Profesionales & $3-1$ & 12.1 \\
\hline $1+2+3$ & 9.4 & 13.7 \\
\hline 4 Técnicos & 6.0 & 5.3 \\
\hline 5 Empleadores administrativos & 7.9 & 4.8 \\
\hline $4+5$ & 13.9 & 5.0 \\
\hline 6 Trabajadores en el comercio & 13.14 & 3.6 \\
\hline 7 Obreros, artesanos, conductores & 25.3 & 3.4 \\
\hline $6+7$ & 38.44 & 3.5 \\
\hline 8 Trabajadores & & \\
\hline de los servicios personales & 14 & 2.2 \\
\hline 9 Trabajadores agrícolas & 19.6 & 1.8 \\
\hline $8+9$ & 34.4 & 2.0 \\
\hline $6+7+8+9$ & 72.84 & 2.8 \\
\hline 10 No clasificados & 3.5 & 4.0 \\
\hline 11 Total & 100.0 & 4.1 \\
\hline
\end{tabular}

Fuente: CEPAL (1999-2000): Panorama social de América Latina.

* Los países son: Brasil, Chile, Colombia, Costa Rica, El Salvador, México, Panamá y Venezuela.

Como puede observarse, el abatimiento arancelario de América Latina y el Caribe ha repercutido directamente y en forma negativa al menos en los dos sectores económicos a los cuales hemos hecho referencia. Esas repercusiones se han dado, como ya se afirmó, en dos dimensiones: en la modificación de la estructura productiva y en el empleo. Ahora bien, si se considera la perspectiva de la distribución ocupacional y los ingresos de los trabajadores del campo y de la ciudad, se puede tener una idea más completa de las condiciones de éstos en el marco de las políticas de apertura.

\section{Estructura ocupacional e ingreso de los trabajadores}

Como puede observarse en el Cuadro 7, en 1997 los trabajadores constituían 73.84\% de la fuerza de trabajo ocupada en los países donde se hizo el estudio y recibieron en promedio un ingreso de 2.8 en líneas de pobreza per cápita. ${ }^{6}$ Dentro de este grupo, los trabajadores

La CEPAL define la línea de pobreza como el "monto de ingreso que permite a cada hogar satisfacer las necesidades esenciales de sus miembros". Entendidas éstas como el "costo de una canasta básica de alimentos 
agrícolas constituyeron casi la quinta parte del total de la fuerza de trabajo y sólo recibieron 1.8 de líneas de pobreza. $\mathrm{Si}$ a estos últimos se le suman los que laboraron en los servicios personales, la cifra alcanzaba un porcentaje de más de $34 \%$ del total con un ingreso promedio de 2 líneas de pobreza.

Los sectores medios constituidos por los técnicos y empleados administrativos que representaron $13.9 \%$ del total percibieron, en promedio, 5 líneas de pobreza, lo que representó menos del doble que el de los trabajadores en general. Lo anterior da idea de un sector débil desde el punto de vista económico y numérico, y sugiere que es resultado de cuando menos dos décadas de aplicación de reformas estructurales.

Los sectores de mayores ingresos representaron apenas 9.4\% de la fuerza de trabajo ocupada con un ingreso promedio de 13.7 líneas de pobreza. Entre éstos, los empleadores, como es natural, se ubicaron en la cúpula de la pirámide. Estos últimos sólo constituyeron 4.3\% del total y tuvieron un ingreso de 15.8 líneas de pobreza.

Si se comparan los ingresos de este último sector con los que obtuvieron en promedio los trabajadores, se observa una diferencia de más de 564\%. Ahora, si se hace la comparación con los trabajadores agrícolas la diferencia es mayor a $877 \%$. Debe señalarse que el estudio no hace diferencia entre empleadores de pequeñas, medianas y grandes empresas, entre los cuales es natural que existan notables desigualdades en términos de ingreso. Estos últimos datos nos ayudan a ver, con mayor precisión, la inequidad en la distribución del ingreso y las repercusiones de las reformas económicas, entre ellas la apertura mediante el desarme arancelario. Ante la perspectiva de su extensión en el esquema del ALCA, existe la preocupación por las modificaciones que pueda sufrir el aparato exportador latinoamericano.

\section{El sector exportador}

La ALADI ha realizado estudios prospectivos sobre el impacto comercial que tendría la eventual puesta en vigor del ALCA. Dichos estudios hacen referencia al comercio intraregional y de las oportunidades de éstos en el mercado norteamericano. En el Cuadro 8 se destacan diez economías latinoamericanas. Como puede observarse, el destino actual de las exportaciones de estas economías está concentrado, en su mayoría, en Estados Unidos, le siguen en importancia la ALADI, la Unión Europea, el MERCosur y, finalmente, el Grupo Andino. Nótese que el estudio no registra exportaciones de estos países hacia Canadá. En el cuadro de referencia se destaca una relativa diversidad en los destinos de las exportaciones de estos países con la notable excepción de México, que las concentra en Estados Unidos. Si se excluye a este último país, el orden del destino de las exportaciones cambia y pasarían

que cubre las necesidades nutricionales de la población, tomando en consideración sus hábitos de consumo, la disponibilidad efectiva de alimentos y sus precios relativos" (CEPAL, 2000). 
Cuadro 8

Retos y oportunidades de las exportaciones de América Latina frente al ALCA

\begin{tabular}{|c|c|c|c|c|c|c|c|c|}
\hline Países & $\begin{array}{r}D e \\
A L A D I\end{array}$ & $\begin{array}{l}\text { stino de } \\
\text { MERCOSUR }\end{array}$ & $\begin{array}{l}l_{E U} a s \\
E U\end{array}$ & $\underset{U E}{e x p o r t}$ & $\begin{array}{c}\text { ciones } \\
\text { Canadá }\end{array}$ & $\begin{array}{l}\% \\
C A N\end{array}$ & $\begin{array}{c}\text { Amenaza de EU y } \\
\text { Canadá a las } \\
\text { exportaciones } \\
\text { regionales } \%\end{array}$ & $\begin{array}{l}\text { Ventaja en } \\
\text { exportaciones } \\
\text { hacia EU y } \\
\text { Canadá \% }\end{array}$ \\
\hline Argentina & 45 & 33 & 10 & 20 & - & - & 25 & 70 \\
\hline Bolivia & 40 & - & 28 & 22 & - & 20 & 25 & 75 \\
\hline Brasil & 25 & 15 & 20 & 30 & - & - & 10 & 60 \\
\hline Chile & 22 & 10 & 17 & 25 & - & - & 10 & 80 \\
\hline Colombia & 20 & - & 40 & 20 & - & 15 & 6 & 75 \\
\hline Ecuador & 20 & - & 40 & 20 & - & 12 & 6 & 90 \\
\hline México & $2 *$ & - & 88 & 4 & - & - & 1 & 75 \\
\hline Paraguay & 55 & 48 & 8 & 32 & - & - & 40 & 85 \\
\hline Uruguay & 55 & 50 & 6 & 18 & - & - & 20 & 75 \\
\hline Venezuela $* *$ & 15 & - & 50 & - & - & 9 & 6 & 80 \\
\hline Promedios & 29.9 & 15.6 & 30.7 & 19.1 & - & 5.6 & 14.9 & 76.5 \\
\hline
\end{tabular}

* Este porcentaje también incluye a países centroamericanos y caribeños.

** 20\% correspondió a los países centroamericanos y caribeños.

Fuente: ALADI (2001).

a primer término las orientadas a la ALADI, seguidas por Estados Unidos, la Unión Europea, el mercosur y el Grupo Andino.

La puesta en práctica del ALCA amenazaría a las exportaciones intraregionales de la ALADI en la medida que implicaría el desplazamiento de $14.9 \%$ de los productos de la región por los provenientes de Canadá y Estados Unidos, en tanto que las oportunidades de acceso al mercado norteamericano y canadiense de los productos provenientes de las economías señaladas sería de $76.5 \%$. En apariencia, estas cifras revelan una ventaja para las economías latinoamericanas. Esta perspectiva tiene dos limitaciones: primero, significaría una reducción en la diversificación que hasta hoy existe del sector exportador de los países latinoamericanos, al menos, de los señalados en el cuadro, lo que se transformaría en una mayor dependencia de sus aparatos económicos. Segundo, si se atiende a las ramas económicas que resultarían beneficiadas - materias primas y alimentos-, se consolidaría la tendencia histórica de asignar a nuestras economías el papel de proveedoras de productos provenientes, principalmente, del sector primario, lo cual ha sido una característica de su atraso.

\section{Conclusiones}

Por lo expuesto, puede afirmarse que la posible instrumentación del ALCA a partir de 2005 impactaría, de forma negativa, la estructura productiva y el comercio exterior de América Latina y el Caribe porque el esquema de integración puede profundizar los procesos de apertura en estos países. En el caso de la producción está la evidencia empírica de los efec- 
tos del desarme arancelario que se puso en práctica en nuestros países a partir de los años ochenta. Dichos efectos han sido amplios. La profundidad de sus consecuencias contribuyó a trastocar negativamente aspectos estructurales de la economía. En este sentido el peso de la pequeña y mediana propiedad en la industria y la agricultura cedió en favor de la grande. Primero, porque la competencia externa provocó el quiebre de miles de pequeñas y medianas industrias y, segundo, por el fortalecimiento de las grandes a través de una mayor incorporación tecnológica en sus procesos productivos. Esto significó, en el plano social, el aumento del desempleo y, en general, del deterioro en la calidad de vida de amplios grupos de población, expresado en el abatimiento del ingreso de los ubicados en la base de la pirámide social.

Respecto al comercio exterior, se prevé que la competencia norteamericana y canadiense modifique el peso del comercio intraregional latinoamericano por la pérdida de mercados de estos últimos, frente a la llegada de los productos procedentes de aquéllos. Consolidaría el papel histórico de países de la región como proveedores de materias primas y alimentos y, en general, modularía el desarrollo desigual de las regiones y países participantes.

\section{Bibliografia}

ALADI, "Impacto del ALCA en el comercio intrarregional y en el comercio con Estados Unidos y Canadá" (varios países), 2001.

CEPAL, El Pacto fiscal: Fortalezas, debilidades, desafíos, Santiago de Chile, 1998, pp. 63-97.

, Panorama Social de América Latina 1999. 2000, Santiago de Chile, 2000, pp. 35-106.

_- Una década de luces y sombras: América Latina y el Caribe en los años noventa, México, 2001, 318 pp.

David, M. Beatriz, César Morales y Mónica Rodríguez, "Modernidad y heterogeneidad: estilo de desarrollo agrícola y rural en América Latina y el Caribe", en Desarrollo rural en América Latina y el Caribe, Bogotá, CEPAL y Alfaomega, 2001, pp. 41-88.

Estévez, Jaime, "Crisis mundial y proyecto nacional", en Pablo González Casanova y Héctor Aguilar Camín (coordinadores), México ante la crisis, México, Siglo xxI, 1985, pp. 45-63.

Ferrer, Aldo, De Cristóbal Colón a Internet: América Latina y la globalización, Buenos Aires, FCE, 2001, pp. 62-79.
Grien, Raúl, La integración económica como alternativa inédita para América Latina, México, FCE, 1994, pp. 154-228, 494-522.

Katz, Jorge, "Cambios estructurales y evolución de la productividad laboral en la industria latinoamericana en el período 1970-1996", Santiago de Chile, CEPAL, 1999 (Serie: Reformas Económicas núm. 14), 48 pp.

__ "Cambios en la estructura y comportamiento del aparato productivo latinoamericano en los años 1990: después del 'Consenso de Washington', ¿qué?”, Santiago de Chile CEPAL, (Serie: Desarrollo productivo núm. 65), 55 pp.

Moguillansky, Graciela y Ricardo Bielschowsky, Inversión y reformas económicas en América Latina, Santiago de Chile, CEPAL-FCE, 2000, 218 pp.

Ocampo, José Antonio, "Agricultura y desarrollo rural en América Latina” en Desarrollo rural en América Latina y el Caribe, Bogotá, CEPAL y Alfaomega, 2001, pp. 1-40.

Peres, Wilson y Giovanni Stumpo, Pequeñas y medianas empresas industriales en América Latina y el Caribe, México, CEPAL-Siglo XXI, 2002, 549 pp. 
Rocha, Carlos H. y Hélio E. Da Silva, "Situación y políticas para pequeñas y medianas empresas industriales en Brasil", en Wilson Peres y Giovanni Stumpo, Pequeñas y medianas empresas industriales en América Latina y el Caribe, México, CEPAL-Siglo XXI, 2002, pp. 79-117.

Romaniello, Gabriela, "Situación actual y política para las pequeñas y medianas empresas industriales en Uruguay", en Wilson Peres y Giovanni Stumpo, Pequeñas y medianas empresas industriales en América Latina y el Caribe, México, CEPAL-Siglo XxI, 2002, pp. 469-509.

Spoor, Max, "Incidencia de dos décadas de ajustes en el desarrollo agrícola de América Latina y el Caribe", en Desarrollo rural en América Latina y el Caribe, Bogotá, CEPAL y Alfaomega, 2001, pp. 135-164.
Stallings, Bárbara y Wilson Peres, Crecimiento, empleo y equidad: el impacto de las reformas económicas en América Latina y el Caribe, Santiago de Chile, CEPAL-FCE, 2000, 302 pp.

Sunkel, Osvaldo y Pedro Paz, El subdesarrollo latinoamericano y la teoría del desarrollo, México, Siglo XXI, 1978, pp. 297-385.

Villarreal, René, La contrarrevolución monetarista (Teoría, política económica e ideología del neoliberalismo), México, Océano, 1984, pp. 23-126.

Weller, Jürgen, Reformas económicas, crecimiento y empleo: los mercados de trabajo en América Latina y el Caribe, Santiago de Chile, CEPAL y FCE, 2000, p. 261.

Yergin, Daniel y Joseph Stanislaw, Pioneros y líderes de la globalización, Buenos Aires, Vergara, 1999. pp. 349-396. 